\title{
Primeiro registro de Acestrorhynchus pantaneiro Menezes, 1992 (Characiformes, Acestrorhynchidae) no sistema da laguna dos Patos, Rio Grande do Sul, Brasil
}

\author{
Adriana Saccol-Pereira ${ }^{1}$, Paulo César Carvalho Milani ${ }^{2}$ e Clarice Bernhardt Fialho ${ }^{1}$
}

Biota Neotropica v6 (n3) -http://www.biotaneotropica.org.br/v6n3/pt/abstract?short-communication+bn00706032006

Recebido em 20/09/05.

Versão reformulada 09/07/06.

Publicado em 01/09/06

\footnotetext{
${ }^{1}$ Laboratório de Ictiologia, Departamento de Zoologia, Instituto de Biociências, Universidade Federal do Rio Grande do Sul (UFRGS). Av. Bento Gonçalves 9500, CEP 91501-970, Porto Alegre, RS, Brasil - www.ufrgs.br/zoologia/index.htm. Autor para correspondência. E-mail: adrisaccol2000@yahoo.com.br.

${ }^{2}$ Laboratório de Dinâmica Populacional, Faculdade de Biociências, Pontifícia Universidade Católica do Rio Grande do Sul (PUCRS). Av. Ipiranga 6681, CEP: 90619-900, Porto Alegre, RS, Brasil - www.pucrs.br/fabio/pos/zoo.
}

\begin{abstract}
Saccol-Pereira, A.; Milani, P.C.C. and Fialho, C.B. First record of Acestrorhynchus pantaneiro Menezes, 1992 (CHARACIFORMES, ACESTRORHYNCHIDAE) in the system of the laguna dos Patos, Rio Grande do Sul, Brazil. Biota Neotrop. Sep/Dec 2006 vol. 6, no. 3 http://www.biotaneotropica.org.br/v6n3/pt/abstract?shortcommunication+bn00706032006 ISSN 1676-0603
\end{abstract}

Acestrorhynchus pantaneiro Menezes, 1992, belonging species in the basins of the Paraguay, lower Paraná, La Plata, and Uruguay rivers is registered in the Parque Estadual Delta do Jacuí, basin of the laguna dos Patos.

Key words: Acestrorhynchus pantaneiro, Acestrorhynchidae, fish, new record.

\section{Resumo}

Saccol-Pereira, A.; Milani, P.C.C. and Fialho, C.B. Primeiro registro de Acestrorhynchus pantaneiro Menezes, 1992 (CHARACIFORMES, ACESTRORHYNCHIDAE) no sistema da laguna dos Patos, Rio Grande do Sul, Brasil. Biota Neotrop. Sep/Dec 2006 vol. 6, no. 3 http://www.biotaneotropica.org.br/v6n3/pt/abstract?shortcommunication+bn00706032006 ISSN 1676-0603

Acestrorhynchus pantaneiro Menezes, 1992, espécie pertencente às bacias dos rios Paraguai, baixo Paraná, La Plata e Uruguai é registrada no Parque Estadual Delta do Jacuí, bacia da laguna dos Patos.

Palavras-chave: Acestrorhynchus pantaneiro, Acestrorhynchidae, peixes, nova ocorrência.

http://www.biotaneotropica.org.br 


\section{Introdução}

A família Acestrorhynchidae apresenta apenas o gênero Acestrorhynchus, dividido em 17 espécies. As espécies da família ocupam uma variedade de hábitats, preferencialmente ambientes lênticos. Apresentam distribuição restrita à América do Sul com maior diversidade específica ocorrendo nas bacias do Amazonas e do Orinoco, além das drenagens do rio São Francisco, Paraná, Paraguai e La Plata.

O gênero Acestrorhynchus pertence a uma tribo homogênea, sendo que a maioria das espécies pode ser identificada por várias características externas, inclusive comprimento de focinho, coloração e tamanho máximo. Todas as formas são ictiófagas e requerem água oxigenada e clara com uma temperatura entre $23-28^{\circ} \mathrm{C}$ (Menezes 1992).

Acestrorhynchus pantaneiro Menezes, 1992, é a única espécie com distribuição no Rio Grande do Sul, e restringese à bacia do rio Uruguai (Menezes 2003). Conhecido popularmente como peixe cachorro, apresenta dentição peculiar e hábito carnívoro. É uma espécie de porte médio apresentando comprimento máximo total de $24 \mathrm{~cm}$ (Oyakawa 2005). Segundo Zaniboni et al. (2004), para o alto rio Uruguai, o comprimento do menor indivíduo capturado foi $6 \mathrm{~cm}$ e o maior 35,2 cm, com pesos de 1,0 e 396,0 gr respectivamente.

A presença de Acestrorhynchus pantaneiro na laguna dos Patos a caracteriza como uma espécie alóctone (IBAMA, Portaria 145/1998), isto é, espécie nativa de bacia hidrográfica brasileira e registrada em bacia onde não ocorreria naturalmente.

O objetivo deste trabalho é relatar o primeiro registro de ocorrência de A. pantaneiro (figura 1) no sistema hidrográfico da laguna dos Patos, dentro dos limites do Parque Estadual Delta do Jacuí - RS, onde foram coletados três exemplares em diferentes meses e locais de coleta.

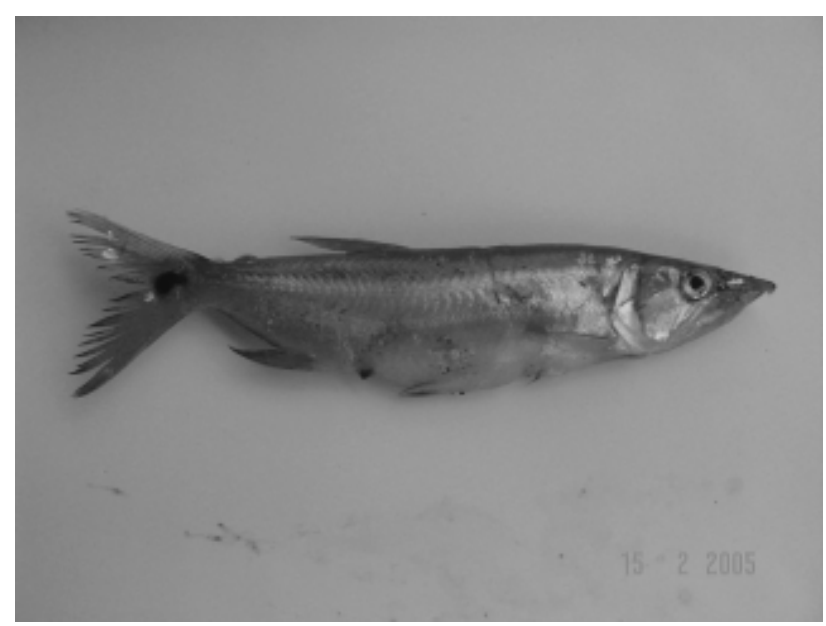

Figura 1: Exemplar de Acestrorhynchus pantaneiro coletado no Parque Estadual Delta do Jacuí - RS.

Figure 1: Specimens of Acestrorhynchus pantaneiro collected in the Parque Estadual Delta do Jacuí - RS.

\section{Material e Métodos}

O Parque Estadual Delta do Jacuí (29 $53^{\circ}$ ' a $30^{\circ}$ 03's; $51^{\circ} 12^{\prime}$ a $51^{\circ} 27^{\prime} \mathrm{W}$ ) está situado na Região Metropolitana de Porto Alegre e abrange as ilhas de formação sedimentar do arquipélago dos rios Jacuí, Gravataí, Caí e Sinos. Atualmente, conta com uma superfície de 21907,07 hectares, compreendendo terras emersas continentais e 28 ilhas (Oliveira 2002). Por apresentar um grande número de ambientes tais como canais, sacos e banhados formados no interior das ilhas, torna-se um local importante para a manutenção e reprodução de vários organismos aquáticos (Koch et al. 2000).

As amostragens foram realizadas em três diferentes locais do parque (figura 2), conhecidos por Saco do Quilombo (29 $58^{\prime} 31^{\prime \prime}$ S; 51 $1^{\circ} 15^{\prime} 53,2^{\prime \prime}$ W), Saco da Alemoa

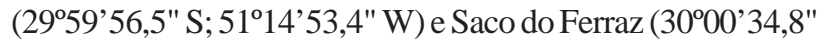
S; 51 $\left.{ }^{\circ} 14^{\prime} 41,3^{\prime \prime} \mathrm{W}\right)$.

Em cada ponto amostral foi utilizada uma bateria de redes de espera com malha de 1,5; 2,5; 3,5; 4,5 e 6,0 cm entrenós adjacentes, dispostas perpendicularmente à margem. Cada rede possuía uma dimensão padronizada de $20 \mathrm{~m}$ de comprimento por $1,5 \mathrm{~m}$ de altura. O esforço de captura foi constante, sendo de 24 horas. As redes foram colocadas às 12:00 h e revisadas às 18:00 $\mathrm{h}$, às 06:00 $\mathrm{h}$ do dia seguinte e às 12:00 $\mathrm{h}$ quando então foram retiradas.

Os indivíduos capturados foram etiquetados e separados de acordo com o ponto, tipo de rede e hora da coleta e fixados em formalina $10 \%$.

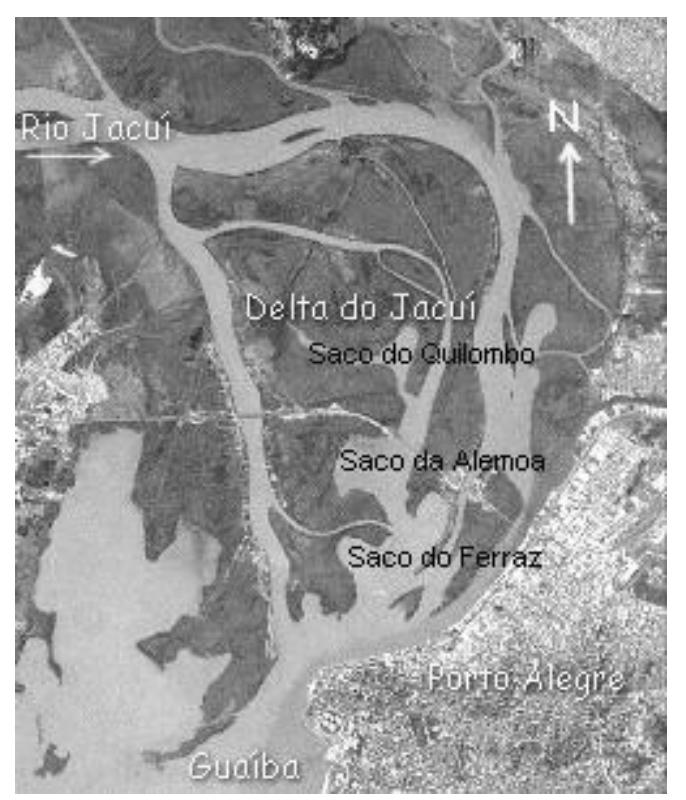

Figura 2: Locais de amostragem no Parque Estadual Delta do Jacuí - RS, Brasil.

Figure 2: Sampling places in the Parque Estadual Delta do Jacuí RS, Brasil.

http://www.biotaneotropica.org.br 
Saccol-Pereira, A.; Milani, P.C.C. and Fialho, C.B. - Biota Neotropica, v6 (n3) - bn00706032006

\section{Resultados}

Todos os A. pantaneiro foram capturados no horário entre 06:00 e 18:00 h, horários da primeira e terceira revisão das redes.

O primeiro exemplar, um macho apresentando comprimento total de $23 \mathrm{~cm}$ e pesando $124,8 \mathrm{~g}$ foi capturado em Novembro de 2004, na localidade conhecida como Saco do Ferraz com rede de emalhe de 2,5 cm entre-nós adjacentes. O segundo indivíduo, uma fêmea apresentando ovócitos com vitelogênese completa (Vazzoler 1996), com comprimento total de $22,5 \mathrm{~cm}$ e pesando $124,5 \mathrm{~g}$ foi coletada em Dezembro de 2004, com rede de emalhe de 2,5 cm entrenós adjacentes em local denominado Saco da Alemoa. O terceiro espécime, um indivíduo jovem, coletado no Saco do Quilombo em Fevereiro de 2005, com rede de emalhe de $1,5 \mathrm{~cm}$ entre-nós adjacentes, apresentou um comprimento total de $16 \mathrm{~cm}$ e peso de 30,3 g. As tabelas 1 e 2 incluem dados morfométricos e merísticos, respectivamente.

As gônadas foram analisadas macroscopicamente baseando-se na classificação proposta por Vazzoler (1996).

Com exceção do indivíduo jovem, cujo estômago estava vazio, macho e fêmea apresentavam estômago cheio, com presença de peixe em seu conteúdo estomacal.

Após dissecação, os exemplares foram fixados em formalina $10 \%$ e tombados na coleção científica do Departamento de Zoologia da Universidade Federal do Rio Grande do Sul, sob os lotes UFRGS 6988, UFRGS 6989 e UFRGS 6990.

Tabela 1: Dados morfométricos (expressos em $\mathrm{mm}$ ).

Table 1: Morphometric data (express in $\mathrm{mm}$ ).

\begin{tabular}{|c|c|c|c|}
\hline Número Coleção & 6988 & 6989 & 6990 \\
\hline Exemplar & macho & fêmea & ind. jovem \\
\hline Compr. Padrão & & & 121.35 \\
\hline Dist. Pré-Dorsal & 192.66 & 188.42 & 79.60 \\
\hline Dist. Pré-Ventral & 117.42 & 119.59 & 88.58 \\
\hline Dist. Pré-Peitoral & 101.46 & 107.25 & 39.98 \\
\hline Dist. Pré-Anal & 55.07 & 52.88 & 91.41 \\
\hline Alt. Corpo & 145.01 & 146.61 & 29.92 \\
\hline Alt. Ped. Caudal & 46.36 & 43.23 & 9.19 \\
\hline Compr. Ped. Caudal & 15.36 & 15.59 & 12.46 \\
\hline Base Nad. Anal & 24.17 & 21 & 28.15 \\
\hline Comp. Nad. Dorsal & 46.79 & 41.83 & 25.33 \\
\hline Comp. Nad. Ventral & 45.62 & 39.31 & 17.02 \\
\hline Comp. Nad. Peitoral & 32.92 & 31.72 & 19.37 \\
\hline Comp. Cabeça & 35.98 & 35.55 & 38.87 \\
\hline Comp. Focinho & 54.15 & 54.5 & 16.10 \\
\hline Comp. Maxilar & 23.61 & 22.1 & 19.87 \\
\hline Diâm. Orb. Olho & 36.80 & 29.5 & 7.23 \\
\hline Larg. Interorbital & 9.72 & 9.65 & 9.65 \\
\hline
\end{tabular}

Tabela 2: Dados merísticos.

Table 2: Meristic data.

\begin{tabular}{|c|c|c|c|}
\hline Número Coleção & 6988 & 6989 & 6990 \\
\hline Exemplar & macho & fêmea & ind. jovem \\
\hline Raios Ram. Anal & $\mathrm{III}+26$ & $\mathrm{III}+24$ & $\mathrm{III}+23$ \\
\hline Raios Dorsal & $\mathrm{II}+7$ & $\mathrm{II}+9$ & $\mathrm{II}+8$ \\
\hline Raios Ventral & $\mathrm{I}+7$ & $\mathrm{I}+6$ & $\mathrm{I}+5$ \\
\hline Raios Peitoral & $\mathrm{I}+16$ & $\mathrm{I}+14$ & $\mathrm{I}+14$ \\
\hline R. Princ. Caudal & $\mathrm{III}+16+\mathrm{III}$ & $\mathrm{III}+17+\mathrm{III}$ & $\mathrm{III}+17+\mathrm{III}$ \\
\hline Série Longitudinal & 116 & 119 & 109 \\
\hline Esc. Linha Lateral & 111 & 99 & 101 \\
\hline Esc. L. Lat. - Nad. Dor. & 25 & 27 & 29 \\
\hline Esc. L. Lat. - Nad. Ven. & 11 & 9 & 10 \\
\hline Dentes Pré-Maxilar & 19 & 17 & 16 \\
\hline Dentes Maxilar Direito & 32 & 30 & 31 \\
\hline Dentes Maxilar Esquer. & 33 & 36 & 30 \\
\hline
\end{tabular}

http://www.biotaneotropica.org.br 


\section{Discussão}

Embora as capturas de A. pantaneiro não apresentem número elevado de exemplares, o fato de uma espécie alóctone encontrar condições propícias para reprodução dentro de um ambiente natural pode proporcionar o estabelecimento da mesma, o que poderia implicar em algum tipo de impacto futuro sobre as populações autóctones. Segundo Araújo (1998), as atividades antrópicas exercem uma profunda e, habitualmente negativa, influência nos peixes de água doce dos menores córregos aos maiores rios. Krebs (2001), relata que a introdução de espécies constitui-se juntamente com a degradação e fragmentação de hábitat, em uma das maiores causas de extinção recente de espécies.

A presença de espécies ocupando bacias que não as suas de origem, como exemplo o Pachyurus bonariensis (Pinto et al. 2001) que atualmente é capturado em escala comercial na lagoa do Casamento, porção nordeste da laguna dos Patos (Milani 2005), vem sendo registradas no Rio Grande do Sul, assim como Clarias gariepinus, espécie exótica capturada na laguna dos Patos (Braun et al. 2003).

Portanto, a presença de A. pantaneiro no sistema hidrográfico da laguna dos Patos pode estar associada ao escape de cultivos em açudes, soltura por pessoas que se dedicam à aquariofilia ou ainda por ação antrópica direta no meio ambiente, modificando os limites hidrográficos de bacias em função socioeconômica, o que, neste caso poderia ter facilitado a transposição da espécie entre as bacias hidrográficas.

\section{Referências Bibliográficas}

ARAÚJO, F.G. 1998. Adaptação do índice de integridade biótica usando a comunidade de peixes para o rio Paraíba do Sul. Rev. Bras. Biol. v.58, n.4, p.547-558.

BRAUN, A.S.; MILANI, P.C.C. \& FONTOURA, N.F. 2003. Registro da introdução de Clarias gariepinus (Siluriformes, Clariidae) na laguna dos Patos. Biocienc., v.11, n.1, p.101-102.

IBAMA (Portaria 145/1998). Instituto Brasileiro do Meio Ambiente e dos Recursos Naturais Renováveis. http:// www.institutohorus.org.br/download/marcos_legais/ PORTARIA N 145 DE 29 DE OUTUBRO DE 1998.pdf. Ultimo acesso em 31/08/2005.

KOCH, W.R.; MILANI, P.C.C. \& GROSSER, K.M. Guia Ilustrado; Peixes Parque Delta do Jacuí. Porto Alegre: Fundação Zoobotânica do Rio Grande do Sul, 2000.

KREBS, C. 2001. Ecology: the experimental analysis of distribution and abundance. 5. ed. Benjamin Cummings, 695p.

MENEZES. N.A. 2003. Família Acestrorhynchidae In Checklist of the Freshwater Fishes of South and Central America (R.E. Reis, S.O. Kullander \& C.J. Ferraris, Jr., eds.). Porto Alegre: EDIPUCRS, Brasil. p.432-433.
MENEZES, N. A. 1992. Redefinição taxonômica das espécies de *Acestrorhynchus* do grupo *lacustris* com a descrição de uma espécie (Osteichthyes, Characiformes, Characidae). Comun. Mus. Ciênc. PUCRS, Sér. Zool. Porto Alegre, p.39-54.

MILANI, P.C.C. 2005. Diagnóstico da pesca artesanal na lagoa do Casamento, sistema nordeste da laguna dos Patos: uma proposta de manejo. Dissertação (Mestrado em Zoologia) - Faculdade de Biociências, PUCRS, Porto Alegre. 71p.

OLIVEIRA, M.L.A.A. 2002. Conhecendo o Parque. In Natureza em Revista, Edição Especial: Delta do Jacuí. Publicação editada pela Fundação de Zoobotânica do Estado do Rio Grande do Sul, p.12-19.

OYAKAWA, O.T. 2005. In Fish base World Wide Web electronic publication (R. Froese and D. Pauly, eds.). www.fishbase.org. Último acesso em 20/08/2005.

PINTO, R.F.; OLIVEIRA, C.L.C.; COLOMBO, P.; MALABARBA, L.R. 2001. Primeiro registro de Pachyurus bonariensis (Steidachner, 1879) (Perciformes, Sciaenidae) para o sistema da laguna dos Patos, Rio Grande do Sul, Brasil. Resumos, XIV Encontro Brasileiro de Ictiologia. São Leopoldo, RS.

VAZZOLER, A.E.M. 1996. Biologia da reprodução de peixes teleósteos: teoria e prática. Maringá: EDUEM. 169p.

ZANIBONI, E.; MEURER, S.; SHIBATTA, O.A.; NUÑER, A.P.O. 2004. Catálogo ilustrado de peixes do alto rio Uruguai. Florianópolis: Ed. da UFSC: Tractebel Energia. 128p.

Título: Primeiro registro de Acestrorhynchus pantaneiro Menezes, 1992 (Characiformes, Acestrorhynchidae) no sistema da laguna dos Patos, Rio Grande do Sul, Brasil

Autores: Saccol-Pereira, A.; Milani, P.C.C. and Fialho, C.B.

Biota Neotropica, Vol. 6 ( número 3): 2006

http://www.biotaneotropica.org.br/v6n3/pt/ abstract?short-communication+bn00706032006

Recebido em 20/09/05 - Versão reformulada 09/07/06. Publicado em 01/09/06

ISSN 1676-0603 